\title{
HUMAN PERFORMANCE ANALYSIS OF INDUSTRIAL RADIOGRAPHY RADIATION
} EXPOSURE EVENTS

\author{
Wendy J. Reece and Susan G. Hill \\ Idaho National Engineering Laboratory \\ Idaho Falls, Idaho
}

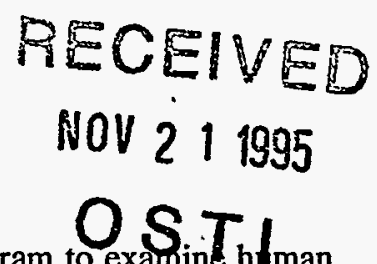

\begin{abstract}
A set of radiation overexposure event reports were reviewed as part of a program to exanin $\mathrm{h}$ man performance in industrial radiography for the U.S. Nuclear Regulatory Commission. Incident records for a seven year period were retrieved from an event database. Ninety-five exposure events were initially categorized and sorted for further analysis. Descriptive models were applied to a subset of severe overexposure events. Modeling included: (1) operational sequence tables to outline the key human actions and interactions with equipment, (2) human reliability event trees, (3) an application of an information processing failures model, and (4) an extrapolated use of the error influences and effects diagram. Results of the modeling analyses provided insights into the industrial radiography task and suggested areas for further action and study to decrease overexposures.
\end{abstract}

\section{INTRODUCTION}

Industrial radiography is the process of using a small gamma radiation source to expose $x$-ray film images of welds and other structural elements, providing a nondestructive means of testing. This type of radiography is used to check the integrity of components in airplanes, pipelines, processing facilities and power plants as well as other structures where visual examination does not provide sufficient data. Detection of structural flaws through the use of radiography can prevent hazardous conditions and the potential for serious accidents (McGuire \& Peabody, 1982).

To perform radiography in the remote industrial setting, a portable crank-out camera device is used. The camera body is internally shielded to safely store the radiation source when not in use. A drive cable is attached at one side of the camera with a connection to the source. On the other side, a guide tube is attached to the camera. The radiographer positions the end of the guide tube at the location to be x-rayed and films are placed appropriately. The task then involves the use of a cranking device at the other end of the drive cable to extend the source out of the camera, through the guide tube to the end of the tube where the radiograph is to be taken. When the exposure is completed, the radiographer must crank the source back into the camera, secure it inside, remove the cable and guide tubes, lock the source in the shielded position, and move the equipment to the next site. Throughout this operation, safety regulations require the use of radiation survey meters. These hand-held devices provide visual indication of the level of radiation in the area. Some meters are also equipped with audible alarms which sound if radiation reaches a threshold level. If the source is left unshielded, the radiographer and other people in the surrounding area may be exposed to unhealthy levels of radiation.

As part of the growing interest in reducing operational risk associated with radiographic materials, the Idaho National Engineering Laboratory (INEL) conducted an evaluation of human performance in industrial radiography overexposure incidents for the U.S. Nuclear Regulatory Commission. The intent of this analysis was to identify the most frequent direct cause(s) of events and to develop recommendations for preventing incidents.

\section{METHOD}

Abstracts and exposure data from the Nuclear Material Events Database (NMED) were used to identify the radiography events (Lipp \& Roberts, 1995). The database contained 124 industrial radiography records collected from across the United States for the period 1987 to 1993. Each record represented an overexposure, with 51 exposures exceeding the regulatory limit. In some cases, several overexposures were related to a single event. Related records were identified, resulting in a final count of 95 events involving some form of reported radiation overexposure,

The availability of additional event information (beyond that captured within the NMED) was limited to the more severe exposure events. Dosimetry badge exposures over time (e.g., quarterly or false readings) and other nonacute incidents are typically not investigated in the same manner as are severe overexposures; therefore the amount of descriptive information for all but the most serious events is limited to brief summaries. For the acute exposure events, additional event-specific information was collected from responsible regulatory entities, such as NRC Regional offices or individual state agencies. A total of 19 events had sufficient descriptive information available for detailed analysis.

To summarize performance, including human errors and equipment failures associated with each of the 19 events, several analysis tools were applied. In order to best capture the information available, each event was examined using four approaches, summarized below.

\section{Operation Sequence Table}

Operation Sequence Tables (OST) (adapted from Meister, 1985) illustrated the sequence of actions taken for each event. The sequence captured who (personnel) or what (equipment) was involved in the event, interactions between individuals and with equipment as well as the relative timing of actions. The OST was used as a basis for the other analyses.

\section{Event Trees}

Event trees are often used as the basis for risk and

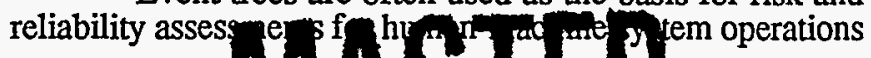




\section{DISCLAIMER}

Portions of this document may be illegible in electronic image products. Images are produced from the best available original document. 
(Gertman \& Blackman, 1994). A generic radiography task event tree was developed to model the various subtasks involved with the start-to-finish task of performing radiography. Each of the 19 overexposure events was diagrammed onto the generic tree to illustrate where within the overall task the errors occurred in each event. A sample section of the generic tree is shown as Figure 1, below.

- Crew fails to inspect equipment

- Crew damages equipment during transport

- Crew fails to lock source in camera

- Crew fails to properly retract source

- Failure of radiation survey to detect exposed source

Figure 1 Sample Section of Radiography Event Tree

\section{Information Processing Failures model}

The Information Processing Failures (IPF) model uses the basic framework of information input, decisionmaking and action execution (O'Hare et al., 1994). Subprocesses that people use to process information are highlighted in the model. This model was included to examine the radiography process in light of the decisionmaking aspect of the task and to provide insights for strategies to modify risky behaviors based on basic learning principles. Table 1 defines the error characterizations that are identified in the IPF model.

\section{Error Influences \& Effects Diagrams}

Error Influences \& Effects Diagrams (as described by Wreathall, 1994) can be used to illustrate human performance and associated performance shaping factors for operational events. These diagrams are rich in information, but detailed event information is required to construct the diagram. To the extent possible, this model was applied to summarize the radiography events overall.

\section{RESULTS}

We reviewed the descriptive modeling of the 19 acute exposure events and identified:

- The kinds of errors recorded (where in the overall radiography task errors are occurring).

- Characterization of the errors (what information processing is associated with the errors).

\section{Relationship Between Characterization and Task Errors}

The information processing failure characterizations for the subtask errors are summarized in Table 2.

As suggested in the descriptive data that we reviewed for each event, equipment set-up errors are primarily characterized by problems with strategy or action. Radiation survey errors, for the most part, are characterized by failure to execute proper procedures. Inspection task errors involve diagnostic difficulties. Scenarios which illustrate the common errors might involve the following elements:

- Set-up resulted in the source being pushed out of the shielded position (e.g., the camera was not locked and the crank handle was bumped, or the camera was moved, during set-up for a shot).

- Set-up resulted in a failure or inability to retract the source (e.g., a kink in the guide tube was not noticed, or the guide tube was positioned with an excessive bend, preventing retraction of the source to the shielded position within the camera).

- Exposure to the source was not detected or responded to by the radiographer due to problems with survey meter usage. This could be due to improper survey, failure to survey, or failure to properly recognize faulty survey equipment.

Table 1 Information Processing Failures Model: Error Characterizations

\begin{tabular}{|c|l|}
\hline Error Characterization & \multicolumn{1}{c|}{ Description } \\
\hline Structural/Mechanical & Equipment failed, no opportunity for radiographer intervention \\
\hline Information Error & Radiographer failed to detect cues arising from change in system status \\
\hline Diagnostic Error & Radiographer failed to accurately diagnose system status, based on available cues \\
\hline Goal Error & Radiographer failed to choose a goal which was reasonable given the circumstances \\
\hline Strategy Error & Radiographer failed to choose an effective strategy to achieve the intended goal \\
\hline Procedure Error & Radiographer failed to properly execute procedures consistent with the selected strategy \\
\hline Action Error & Radiographer failed to physically accomplish the procedure as intended \\
\hline
\end{tabular}


Table 2 Relationship Between Characterization and Task Errors

\begin{tabular}{|c|c|l|}
\hline Task Error & \# Events & \multicolumn{1}{|c|}{ IPF Characterization (\# events) } \\
\hline Equipment set-up & 8 events & Strategy (4), Action (3), Procedure errors (1) \\
\hline No survey/improper survey & 8 events & Procedure (6), Information (1), Diagnostic (1) \\
\hline Equipment inspection & 4 events & Diagnostic (4), Strategy (1) \\
\hline Failure to lock the camera & 3 events & Procedure errors (3) \\
\hline Set-up restricted area & 1 event & Diagnostic error (1) \\
\hline Manual retrieval of source & 1 event & Strategy error (1) \\
\hline
\end{tabular}

\section{Error Characterization}

The five most frequently noted error categories (i.e., procedure, strategy, diagnosis, action, and information) are described below.

Procedure errors occur when proper procedures are not followed. With the development of a routine or habit in performance of the task, proper procedures can be complied with, even without re-reading written procedures during completion of each task. Failure to follow procedures may be unintentional or intentional. If the procedure error is intentional, the procedure cannot be enforced. For example, most modern automobiles are equipped with visual and audible cues to alert the driver to fasten seatbelts. One may choose to ignore the light on the dashboard and the beeping sound, and to not fasten seatbelts. The pieces of information ("cues") provided serve as reminders but cannot ensure or enforce compliance. In radiography, workers may forget to use the survey meter or to lock the camera after each shot. These are examples of procedural errors.

Strategy errors involve the use of an ineffective plan or strategy for accomplishing the goal. Strategy errors are linked to problem solving or troubleshooting-type skills when operators come to wrong conclusions or develop incorrect plans for handling a situation. For example, in an event where the source became disconnected from the drive cable and fell from the camera, the radiographer decided to use his hand to retrieve the source and shove it back into the camera port. A good strategy in this case would have been to set-up and maintain a restricted area while waiting for the radiation safety officer to arrive and retrieve the source properly.

Diagnostic errors involve difficulties in accurately diagnosing system status. Diagnosis relies on an understanding of the system, equipment, and the information provided. For example, when a

radiographer approaches the camera with a survey meter, some low level reading is expected. If the meter reads zero, and the radiographer fails to recognize that a zero reading near the camera is a sign of something wrong with the meter, then a diagnostic error has been made.

Action errors concern the failure to properly execute the intended procedure. This involves the physical motions by the operator that are necessary to complete a procedure. An example of an action error is the incorrect connection of the drive cable to the source assembly by a radiographer trainee. This is a proper step in the procedure of setting up the radiographic equipment, but with the step being performed incorrectly.
Information errors occur when available cues about system status are not clearly received by the operator. Information errors involve human sensory capabilities (e.g., eyesight, hearing), and existing environmental conditions (e.g., adequate lighting, noise level, relative heat or cold). In one event, a radiographer trained to use an alarming ratemeter was working in a high noise area and was wearing ear protection. When his ratemeter sounded an alarm, he could not hear it. This is an example of an information error.

\section{Suggestions for Preventing Radiography Overexposures}

Based on principles of human performance and cognition, we developed suggestions for how errors might be addressed, including:

- Where training may be most effective.

- Where equipment interface enhancements may be most appropriate.

-Where job aids might help performance.

Performance on all types of tasks will improve with experience and practice, given a sufficiently well-designed human-machine interface equipment design. Training can target improvements in areas of the task where procedures, strategy, or diagnosis are involved. Enhanced system/equipment interface design can address diagnostic, information, action, and structural/mechanical difficulties. Job aids that address procedural and diagnostic errors can improve performance. To help illustrate how these measures may be appropriate, the error characterizations are identified below.

Procedure Errors. Training and experience will increase procedural performance. To encourage performance according to procedures, feedback (reminders) can be provided to operators in the form of "job aids." Job aids (e.g., checklists) provide information which is easily understood and helps the worker to complete the task. This is most effective if the information is presented in immediate response to a sequence of events or a particular condition or behavior/action. Equipment design improvements may also help to reduce procedural errors by making the operations easier to complete without variance from the established procedure.

Strategy Errors. The ability to develop efficient strategies is improved with experience. Training can supplement experience by providing practice and feedback on performance in controlled, "simulated" work scenarios. Procedures should address the circumstances under which supervision should be called. 
Diagnostic Errors. Training can improve diagnostic skill, and job aids can assist the workers by providing organized information that doesn't require memorization or recall. Equipment improvements and job aids can also assist in diagnosis of system status, by making the information more readily observed or understood.

Action Errors. Expertise in the execution of actions can be achieved with practice and enhanced with equipment design improvements that consider good human factors principles of human-machine interface design.

Information Errors. With attention to the types of things which may impact performance, equipment can be modified to provide redundancy where needed to overcome environmental interference. For example, visual signals can be provided in addition to audible cues of system status when a noisy environment is anticipated.

\section{In summary:}

- Procedure errors occur in the use of survey meters and in locking the camera after each exposure/retraction of the source to the shielded position. These errors may be effectively addressed with training on the proper use of meters and the routine of retracting the source and locking the camera after each radiograph. Job aids could also be developed to provide additional cues about system status to help workers execute procedures properly. Equipment design can help facilitate worker compliance with procedures by making procedural steps easy to execute.

- Strategy errors are made in equipment set-up and in retrieval of disconnected sources. Training in strategy development could assist workers in formulating efficient strategies for dealing with difficulties in equipment set-up and in accidental source disconnect situations. Procedures should address the use of supervisory personnel for assistance with strategy-related task scenarios. Action errors are also involved with equipment set-up. These can be addressed with hands-on practice in work simulations during training.

- Diagnostic errors occur in inspection of equipment for proper functioning. The data indicate that this is particularly true in the case of survey meters. Training emphasizing how to diagnose meter status could help improve performance. Equipment enhancements, such as a bright light to indicate that the meter is functioning properly would help operators interpret equipment status. Job aids could also be developed to facilitate accurate system diagnosis.

\section{DISCUSSION}

Although the industrial radiography operation involves a fairly simple set of manual tasks, it poses an interesting scenario for human performance analysis. The nature of the routine manual tasks, work schedules, and lack of cues to the operator are particularly important contributors to the overexposure risk associated with industrial radiography. Also of note is the fact that there are no immediate contingencies for risky behaviors (e.g., improper use of survey equipment, physical contact with the radiation source) since, in most cases, radiation exposure has no noticeable instant effect.

Errors in performance of industrial radiography can be characterized by the types of information processing involved in the task. The data we utilized in this study indicated that errors are commonly occurring in the settingup of equipment before the radiograph and in the use of survey meters throughout the process. These errors involve diagnosis of system status, development of work strategies, and the execution of procedures. To address the errors (and the associated potential for radiation overexposures), several methods are suggested, including specialized training, equipment redesign or enhancement, and the use of job aids.

In conducting this evaluation of human performance in industrial radiography, we discovered that data are, for the most part, unavailable. During the period studied, no specific protocol was used by all incident investigators; the data that were collected typically focused upon calculations of personnel exposures and regulatory violations. To understand the human errors involved in the radiography overexposure events, a data collection method is needed to provide a consistent and comprehensive source of information. In the course of this study we noted that an easy-to-use guide for data collection during incident investigations might be useful.

Based upon our understanding of the radiography task and industry practices, we developed a brief questionnaire form to assist incident investigators in collecting human performance data from radiography events. The form includes questions related to the personnel involved in the incident, description of the event, corrective actions, and the investigator's conclusions regarding the event cause and contributing factors.

Investigators will take a copy of the form with them to the event site. Through interviews with the personnel involved (i.e., radiographers, safety officers), the investigators will complete the sections provided on the form, using additional paper as needed. Most questions will have short answers. The back of the form includes the generic radiography task list and space for the investigator to sketch the layout of the event site and perform exposure calculations. The subtasks associated with the incident should be checked on the generic task list. If an additional subtask is involved (i.e., one which is not listed), the investigator should write in a description of the subtask in the space provided.

\section{SUMMARY}

This study identified tasks that were performed incorrectly and characterized those task errors in terms of a simple information processing model. By using this approach, suggested areas for improvement were identified. A data collection form was developed to assist incident investigators in collecting human performance information from radiography radiation overexposure events.

\section{ACKNOWLEDGMENT}

Work supported by the U.S. Nuclear Regulatory Commission under Department of Energy Contract DEAC07-94ID13223. Views expressed in this report do not necessarily reflect the views of the NRC or DOE.

\section{REFERENCES}

Gertman, D.I. and Blackman, H.S. (1994) Human Reliability \& Safety Analysis Data Handbook. New York: Wiley.

Lipp, D.R. and Roberts, G.D. (1995) Nuclear Materials Events Database Coder's Manual, Revision 6. Idaho Falls ID: Idaho National Engineering Laboratory. 
McGuire, S.A. and Peabody, C.A. (1982) Working Safely in Gamma Radiography. (NUREG/BR-0024). Washington D.C.: U.S. Nuclear Regulatory Commission.

Meister, D. (1985) Behavioral Analysis and Measurement Methods. NY: Wiley.
O'Hare, D. et al (1994) Cognitive failure analysis for aircraft accident investigation. Ergonomics, 37(11), - 1855-1869.

Wreathall, J. (1994) Review of medical misadministration event summaries and comparison of human error modeling. (Technical report) Dublin, OH: J. Wreathall \& Company Inc.

\section{DISCLAIMER}

This report was prepared as an account of work sponsored by an agency of the United States Government. Neither the United States Government nor any agency thereof, nor any of their employees, makes any warranty, express or implied, or assumes any legal liability or responsibility for the accuracy, completeness, or usefulness of any information, apparatus, product, or process disclosed, or represents that its use would not infringe privately owned rights. Reference herein to any specific commercial product, process, or service by trade name, trademark, manufacturer, or otherwise does not necessarily constitute or imply its endorsement, recommendation, or favoring by the United States Government or any agency thereof. The views and opinions of authors expressed herein do not necessarily state or reflect those of the United States Government or any agency thereof. 